\title{
Use acupuncture to relieve perimenopausal syndrome: study protocol of a randomized controlled trial
}

Ying Li ${ }^{1}$, Hui Zheng ${ }^{1}$, Qianhua Zheng ${ }^{1}$, Ling Zhao ${ }^{1}$, Erqi Qin ${ }^{1}$, Yu Wang ${ }^{1,2}$, Qian Zeng ${ }^{3}$, Huabin Zheng ${ }^{3}$, Yu Zhao ${ }^{4}$, Wei Sun ${ }^{1}$, Xiaoxia Zhang ${ }^{1}$, Zhishun Liu ${ }^{5}$ and Baoyan Liu ${ }^{5^{*}}$

\begin{abstract}
Background: Whether acupuncture is effective for relieving perimenopausal syndrome has been controversial recently. In this article, we report the protocol of a randomized controlled trial using acupuncture to treat perimenopausal syndrome, aiming to answer this controversy.

Design: A multicenter randomized controlled trial with two parallel arms is underway in China. Two hundred and six women with perimenopausal syndrome will be randomly assigned to a treatment group using acupuncture plus auricular acupressure (AA group) and a control group using Climen ${ }^{\circledast}$ (Bayer Healthcare Company Limited, Guangzhou, China), a 28-day sequential hormone replacement therapy, in a 1:1 ratio. Participants in the AA group will receive three acupuncture sessions per week in the first 4 weeks and two sessions per week in the following 8 weeks, for a total of 28 sessions over 12 weeks. Auricular points will be plastered by Semen Vaccariae twice per week for a consecutive 12 weeks, with both ears used alternately. The Climen ${ }^{\oplus}$ control group is prescribed a tablet containing estradiol valerate $2 \mathrm{mg} /$ day for the first 11 days, and a tablet containing estradiol valerate $2 \mathrm{mg} /$ day plus cyroterone acetate $1 \mathrm{mg} /$ day for the following 10 days. The total treatment period of the control group is three cycles. The post-treatment follow-up period will last 24 weeks. The primary outcome is the Menopause Rating Scale (MRS) assessed at baseline and 4, 12, 16, 24 and 36 weeks after randomization. The secondary outcomes are Menopause-Specific Quality of Life, average hot flash score during 24 hours, serum estradiol, follicle-stimulating hormone and luteinizing hormone level. The first two secondary outcomes are measured at the same point as the MRS. Other secondary outcomes are measured at baseline and 12, 24 weeks after randomization.
\end{abstract}

Discussion: The results of this trial, which will be available in 2015, will clarify whether acupuncture is effective to relieve perimenopausal syndrome.

Trial registration: ClinicalTrials.gov NCT01933204 (registered 9 August 2013)

Keywords: acupuncture, randomized controlled trial, perimenopausal syndrome, study protocol

\section{Background}

Perimenopause is defined as a period during 2 to 8 years preceding menopause and 1 year after final menstruation [1]. During this period, the changes in serum hormone levels - especially estrogen - cause perimenopausal syndrome, manifested as menstrual irregularities, hot flashes, sweats, insomnia, emotional disorders,

\footnotetext{
* Correspondence: baoyanliutcm@sina.com

${ }^{5}$ Guang'anmen Hospital, China Academy of Chinese Medical Sciences, No. 5 BeiXianGe Street, XiCheng District, Beijing 100053, PR China

Full list of author information is available at the end of the article
}

vaginal dryness, sexual dysfunction, and so forth. The syndrome significantly reduces the women's healthrelated quality of life [2,3]. Hormone replacement therapy (HRT) is an effective method to relieve these symptoms [4]. According to recent studies, however, HRT may increase the risk of coronary heart disease [5], stroke [6], breast cancer [7], and endometrial cancer [8]. The benefits of estrogen plus progestin are unable to counterbalance the risks, indicated by the Women's Health Initiative [9]. More and more women therefore 
seek alternative therapies rather than HRT, because of the life-threatening side effects $[10,11]$.

As one of the complementary and alternative therapies without serious side effects [12,13], acupuncture is used frequently to treat a wide range of health problems [14], including hot flashes, which is one of the most common symptoms and the reason why women in the stage of menopausal transition seek medical care [15]. However, several randomized controlled trials (RCTs) indicated that acupuncture is not superior to sham acupuncture in relieving perimenopausal symptoms [16-19]. Reliable and valid evidence for the effectiveness of treating perimenopausal syndrome with acupuncture is still inadequate, due to the number, size, design scheme, and quality of recent studies. We therefore designed a multicenter RCT to address these problems, anticipating providing a more conclusive answer to these questions.

In this trial we primarily aim at investigating whether acupuncture is effective and safe in treating perimenopausal syndrome through a comparison with HRT. This work reported in this article is financed by the National Key Technology R\&D Program during the Twelfth Five- year Plan Period of China, and is registered with identifier NCT01933204 by ClinicalTrials.gov in the USA.

\section{Method and design \\ Study design}

This study is a multicenter RCT comparing an acupuncture plus auricular acupressure (AA) treatment group with a Climen ${ }^{\circ}$ (Bayer Healthcare Company Limited, Guangzhou, China) control group (Figure 1, Table 1). Two hundred and six participants will be included from the following three hospitals in Chengdu, Sichuan, China: First Affiliated Hospital of Chengdu University of Traditional Chinese Medicine (TCM), Chengdu Integrated TCM \& Western Medicine Hospital, and General Hospital of Chengdu Military Region of the Chinese People's Liberation Army.

These participants will be randomly assigned to two groups (AA treatment group and Climen ${ }^{\circ}$ control group) through central randomization in a 1:1 ratio. The central randomization system will be used and performed by the Clinical Evaluation Center of China Academy of Chinese

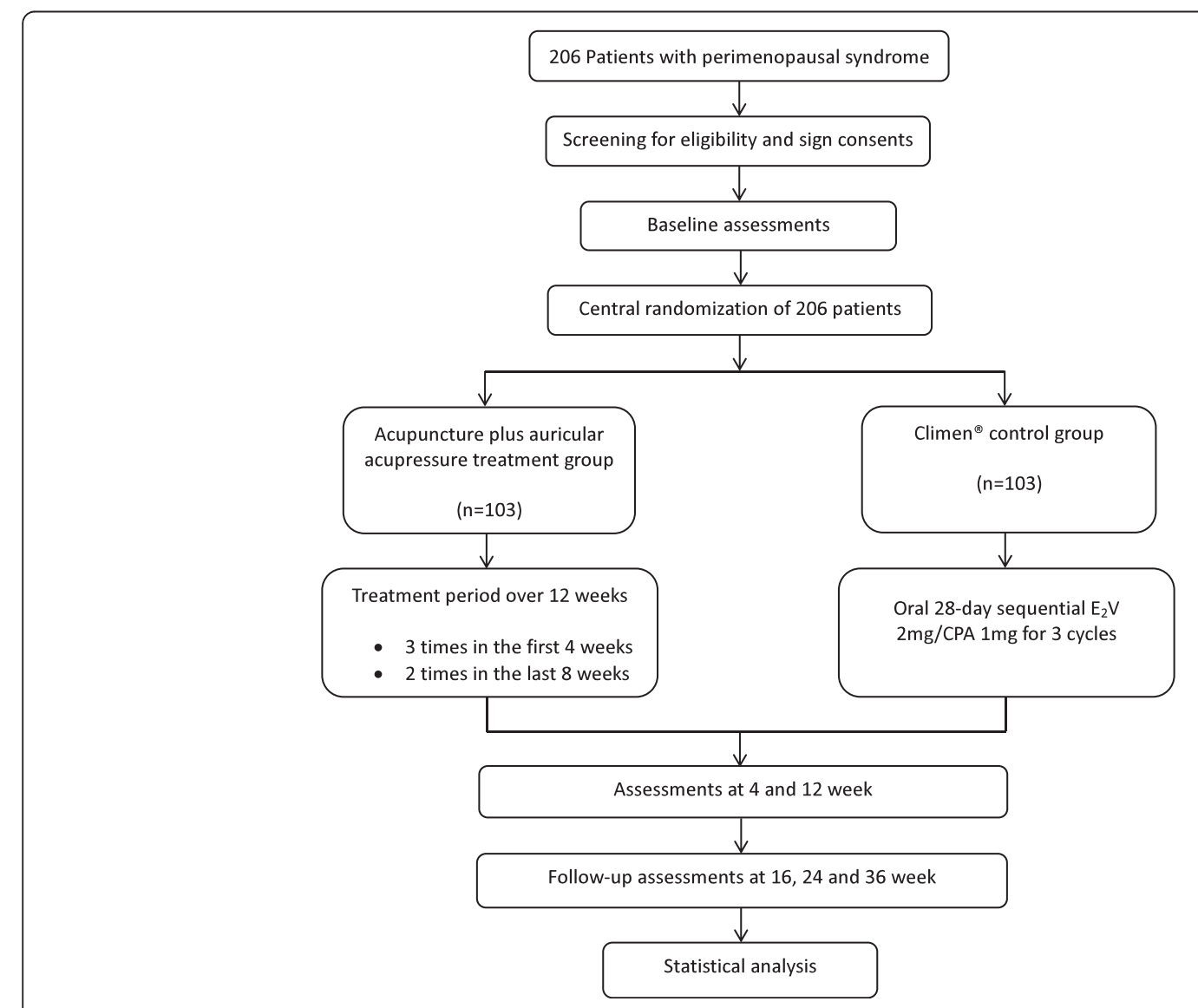

Figure 1 Trial flow chart. CPA, cyroterone acetate; $E_{2} V$, estradiol valerate. Climen ${ }^{\circledast}$ from Bayer Healthcare Company Limited (Guangzhou, China). 
Table 1 Trial processes chart

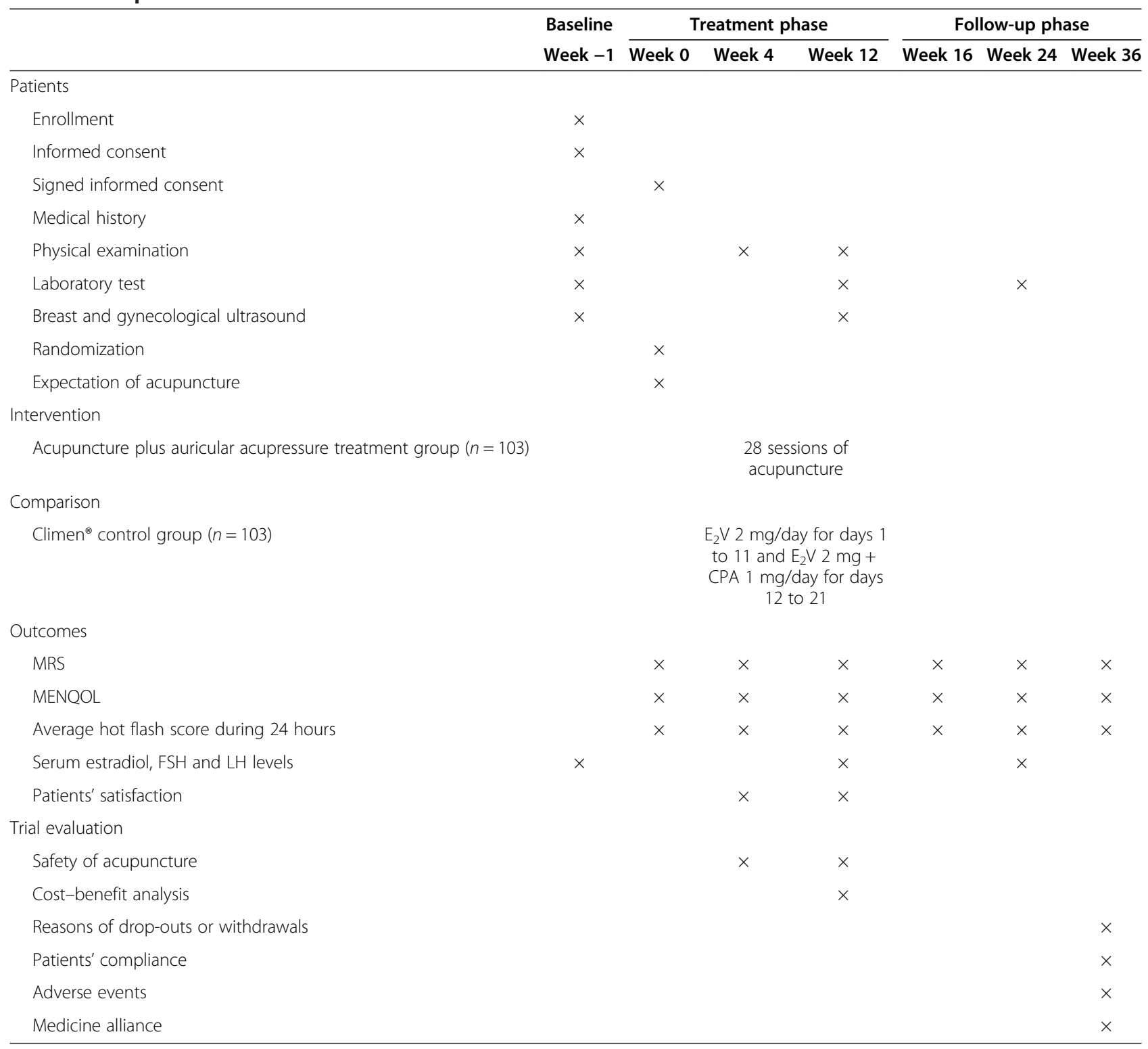

CPA, cyroterone acetate; $E_{2} V$, estradiol valerate; FSH, follicle-stimulating hormone; LH, luteinizing hormone; MRS, Menopause Rating Scale; MENQOL, Menopause-Specific Quality of Life. Climen ${ }^{\circledR}$ from Bayer Healthcare Company Limited (Guangzhou, China).

Medical Sciences in Beijing. Authorized researchers can obtain random numbers and group assignments by calling the central randomization service number or logging into the website to apply. This procedure assures that randomization will not be influenced either by the acupuncturist or the patients.

The total observation period within this study is 37 weeks, including 1 week of baseline, 12 weeks of treatment and 24 weeks of follow-up. After randomization, participants will receive 28 sessions of acupuncture over a period of 12 weeks plus auricular acupressure or take Climen ${ }^{\circledR}$ (from Bayer Healthcare Company Limited, Guangzhou, China) tablets for three cycles. Acupuncture will be given as three sessions weekly in the first 4 weeks and two sessions weekly in the following 8 weeks for each patient. Each session will last 30 minutes. The follow-up period will last 24 weeks (13 to 36 weeks after randomization). Patients will be assessed before randomization as well as 4, 12, 16, 24 and 36 weeks after randomization.

\section{Ethics}

This study was approved by Sichuan Regional Ethics Review of Committee on Traditional Chinese Medicine in June 2013 (No. 2013KL-013), and follows the principles of the Declaration of Helsinki (Version Edinburgh 2000). All participates are asked to sign the written 
informed consent before randomization. All patients will be informed and given enough time to decide whether they would sign the informed consent and participate in this trial or they will be given other treatment options if they are not willing to.

\section{Patients \\ Study population and sample size}

This study focuses on women experiencing perimenopausal syndrome. According to the previous study by Guang'anmen Hospital, China Academy of Chinese Medical Sciences (results still unpublished), the improvement of Menopause Rating Scale (MRS) scores of the electro-acupuncture group and the sham acupuncture group were 6.5 and 2 , respectively. The difference in scores between the two groups was 4.5 , and electroacupuncture was not superior to sham acupuncture. Accordingly, we assume that a difference within 4.5 indicates acupuncture is not inferior to Climen ${ }^{\circ}$ treatment. Using a non-inferiority design, and assuming a power of $90 \%$, an alpha value of $5 \%$, and a population standard deviation of 10, a total of 206 patients will be needed in this study. Each group needs 103 patients (assuming a $20 \%$ dropout rate).

\section{Inclusion criteria}

According to the 2011 criteria of the International Menopause Society [20] and the 2001 criteria of the North American Menopause Society [21], women aged between 40 and 55 who meet the following conditions (Table 2) will be included in this study: menstrual irregularity in the past 12 months, or menstrual period off at least twice in the past 12 months, or amenorrhea 2 to 12 months; suffering any of the following symptoms vasomotor symptoms (hot flashes, sweats), psychological symptoms (insomnia, migraine, irritability), or genitourinary symptoms (vaginal dryness, dyspareunia); serum follicle-stimulating hormone levels $>10$ units/L, or decline of estradiol after amenorrhea; and voluntarily participating in this study with a written informed consent form signed by themselves.

\section{Exclusion criteria}

Patients with any of the following conditions (Table 2) will be excluded: non-natural menopause, due to surgery (for example, hysterectomy with ovarian conservation, and hysterectomy with bilateral oophorectomy; because the severity or patterns of symptoms may differ from the natural menopause, we therefore exclude these participants [22,23]), radiation, or medication; use of HRT, selective serotonin reuptake inhibitors, vitamin $\mathrm{E}$ or black cohosh (which was reported may alleviate menopausal symptoms [24]) in the past 4 weeks; any contraindication of HRT (for example, family history of breast cancer, hysteromyoma, benign galactophore diseases, or ovarian cyst); unknown causes of vaginal hemorrhage; poorly controlled hypertension, diabetes mellitus; history of malignant cancer, mental disorders (including depression),

Table 2 Eligibility criteria

\begin{tabular}{|c|c|}
\hline Inclusion criteria & Exclusion criteria \\
\hline - 40 to 55 years old & - Non-natural menopause (due to surgery, radiation, medication) \\
\hline $\begin{array}{l}\text { - Perimenopausal female (menstrual irregularity in past } 12 \text { months; } \\
\text { or menstrual period off at least twice in past } 12 \text { months; or amenorrhea } \\
\text { of } 2 \text { to } 12 \text { months) }\end{array}$ & - Use of HRT, SSRIs, vitamin E, or black cohosh in past 4 weeks \\
\hline $\begin{array}{l}\text { - Suffering any following perimenopausal symptoms: vasomotor symptoms } \\
\text { (hot flashes, sweats), psychological symptoms (insomnia, migraine, } \\
\text { irritability), or genitourinary symptoms (vaginal dryness, dyspareunia) }\end{array}$ & $\begin{array}{l}\text { - Any contraindication of HRT (for example, family history of breast } \\
\text { cancer, hysteromyoma, benign galactophore diseases, or ovarian cyst) }\end{array}$ \\
\hline - Serum FSH level >10 units/L, or decline of estradiol after amenorrhea & - Unknown causes of vaginal hemorrhage \\
\hline \multirow[t]{9}{*}{ - Willing to receive treatment and sign consents } & - Poorly controlled hypertension, diabetes mellitus \\
\hline & $\begin{array}{l}\text { - History of malignant cancer, mental disorders (including depression), } \\
\text { thyroid diseases, diabetic neuropathy }\end{array}$ \\
\hline & - Dermatosis (for example, eczema, psoriasis) \\
\hline & $\begin{array}{l}\text { - Coagulation defeats or use of anticoagulant medicine } \\
\text { (for example, warfarin, heparin, and so forth) }\end{array}$ \\
\hline & - Use of sedative or anti-anxiety drugs \\
\hline & - Cigarette smoking, alcohol or drug abuse \\
\hline & - Pacemaker or artificial joints \\
\hline & - Pregnant women or women in lactation \\
\hline & - Participation in other clinical trials \\
\hline
\end{tabular}


thyroid diseases, diabetic neuropathy; dermatosis (for example, eczema, psoriasis); any serious diseases of the heart, liver, or kidney (there may be risks that acupuncture induces stress action and thus harms patients with serious illness); coagulation defeats or use of anticoagulant medicine (for example, warfarin, heparin, and so forth - the acupuncture procedure involves penetration of the skin, we therefore will exclude participants with bleeding problems); use of sedative or anti-anxiety drugs; cigarette smoking, alcohol or drug abuse; artificial joints or heart pacemaker; pregnant women or women in lactation (it is still unclear whether acupuncture will lead to abortion or other side effects); and participation in other clinical trials.

\section{Recruitment procedures}

Three strategies will be used to recruit participants with perimenopausal syndrome. The first is to recruit participants in outpatient clinics from the three hospitals. The chief physicians from the departments of gynecology and acupuncture in each hospital were invited to attend a discussion of how to recruit patients. Research assistants will be sent to their departments to help screening participants. Moreover, posters of this trial will be shown outside the clinics to attract possible candidates. Second, we will post advertisements through television broadcasts, newspapers, and so on. In these advertisements, we will briefly introduce the population we want to include, and mention the free treatment for participants who are eligible. Third, we will organize several education groups for women experiencing the perimenopausal period; introducing these people to some basic knowledge and healthcare, as well as this trial in order to attract possible patients who want to participate.

\section{Interventions and comparison \\ Rationale for acupuncture and auricular acupressure protocol}

The acupuncture plus auricular acupressure protocol in this trial was formed in two steps: first, we screen for a range of acupuncture points, auricular points, and stimulation methods through a systematic review of record on ancient books and acupuncture textbooks, as well as results of published articles [25]. Second, meetings were held to further screen for a standardized acupuncture protocol and consensus reached among acupuncture experts in China. Based on the theory of acupuncture and TCM, perimenopausal syndrome is caused by kidney deficiency, exhaustion of Tiangui (sex promoters), deficiency of essence and blood, depletion of the Chong and Ren meridians, imbalance between Yin and Yang of the kidney, and dysfunction of the viscera. According to TCM differentiation, perimenopausal syndrome corresponds to the main pattern of kidney Yin deficiency, kidney Yang deficiency, kidney
Yin and Yang deficiency, liver and kidney deficiency, disharmony between heart and kidney, and spleen and kidney Yang deficiency [26,27]. We therefore chose Guanyuan (RN4), Zigong (EX-CA1), Zusanli (ST36), and Sanyinjiao (SP6) as the primary acupuncture points. In addition, subsidiary acupoints based on the different symptoms of each patient are Hegu (LI4) and Fuliu (KI7) for hot flashes and sweats; Baihui (DU20) and Anmian for sleep disorders; Taichong (LR3) and Shenmen (HT7) for mood disorders; Fengchi (GB20) and Tinggong (SI19) for dizziness and tinnitus; Ligou (LR5) and Taixi (KI3) for vaginal dryness and painful intercourse; and Xuanzhong (GB39) and Yanglingquan (GB34) for aching bones. All of the acupoints chosen above, except Guanyuan (RN4) and Baihui (DU20), which are located in the middle of line, are needled bilaterally. According to the theory of TCM, we also choose auricular points as follows: heart $\left(\mathrm{CO}_{15}\right)$, liver $\left(\mathrm{CO}_{12}\right)$, kidney $\left(\mathrm{CO}_{10}\right)$, spleen $\left(\mathrm{CO}_{13}\right)$, endocrine $\left(\mathrm{CO}_{18}\right)$, internal genital $\left(\mathrm{TF}_{2}\right)$, subcortex $\left(\mathrm{AT}_{4}\right)$, Er Shenmen $\left(\mathrm{TF}_{4}\right)$, sympathetic $\left(\mathrm{AH}_{6 \mathrm{a}}\right)$, and apex of antitragus $\left(\mathrm{AT}_{1,2,4 \mathrm{i}}\right)$ (Table 3).

\section{Acupuncture plus auricular acupressure treatment group}

All acupoints are punctured by filiform needles when patients are lying in a comfortable supine position. Sterile, disposable Hwato needles (provided by Suzhou Hwato Medical Instruments Co. Ltd, Suzhou, China) are used in this study; they are 25 to $50 \mathrm{~mm}$ in length and $0.30 \mathrm{~mm}$ in diameter. The needle is inserted with the

Table 3 Acupuncture points and auricular points

\begin{tabular}{|c|c|c|}
\hline Point & Primary & Additional \\
\hline \multirow[t]{6}{*}{ Acupuncture } & Guanyuan (RN4) & Hegu (LI4), Fuliu (KI7) \\
\hline & Zigong (EX-CA1) & Baihui (DU20), Anmian \\
\hline & Zusanli (ST36) & Taichong (LR3), Shenmen(HT7) \\
\hline & Sanyinjiao (SP6) & Fengchi (GB20), Tinggong (SI19) \\
\hline & & Ligou (LR5), Taixi (KI3) \\
\hline & & $\begin{array}{l}\text { Xuanzhong (GB39), } \\
\text { Yanglingquan (GB34) }\end{array}$ \\
\hline \multirow[t]{10}{*}{ Auricular } & Heart $\left(\mathrm{CO}_{15}\right)$ & \\
\hline & Liver $\left(\mathrm{CO}_{12}\right)$ & \\
\hline & Kidney $\left(\mathrm{CO}_{10}\right)$ & \\
\hline & Spleen $\left(\mathrm{CO}_{13}\right)$ & \\
\hline & Endocrine $\left(\mathrm{CO}_{18}\right)$ & \\
\hline & Internal genital $\left(\mathrm{TF}_{2}\right)$ & \\
\hline & Subcortex $\left(A T_{4}\right)$ & \\
\hline & Er Shenmen $\left(\mathrm{TF}_{4}\right)$ & \\
\hline & Sympathetic $\left(\mathrm{AH}_{6 \mathrm{a}}\right)$ & \\
\hline & $\begin{array}{l}\text { Apex of antitragus } \\
\left(\mathrm{AT}_{1,2,4 i}\right)\end{array}$ & \\
\hline
\end{tabular}


double hand-needle insertion technique. The depth of insertion is adjusted based on the standard permissible depth of insertion for each acupoint. The needle is then twirled, rotated, lifted and thrust moderately until achieving De qi sensation. The needles are retained for 30 minutes in every session and manipulated twice every 10 minutes with intermittent stimulation. Each manipulation lasts for 10 seconds. All needles are taken out with clean cotton balls to avoid bleeding after 30 minutes. Every patient will receive 28 sessions of acupuncture in total over a period of 12 weeks: three sessions per week in the first 4 weeks, every 2 days with a 1-day interval; and two sessions per week in the following 8 weeks, every 3 days with a 2-day interval.

All of the auricular points will use plaster therapy. One piece of Semen Vaccariae is put in the middle of one piece of $0.5 \mathrm{~cm} \times 0.5 \mathrm{~cm}$ medical adhesive plaster. After sterilizing the skin of the auricular points, the plasters are stuck to the points with hemostatic forceps. The patients are required to press the auricular points gently by themselves three to five times a day lasting 1 minute, especially pressing them half an hour before sleep for 2 minutes, until the patients have distention, soreness, numbness, and warm sensation with the local auricular skin congestive, flashed and hot. The strength of pressing should consist of the individual's endurance. The plaster with Semen Vaccariae is exchanged for a fresh set twice weekly for 12 consecutive weeks, every 3 days with a 2-day interval, both ears used alternately.

\section{Climen $^{\circledast}$ control group}

The patients are prescribed Climen (complex packing estradiol valerate with cyroterone acetate tablets; Bayer Healthcare Company Limited) for three cycles: a 28-day sequential therapy combing estradiol valerate $2 \mathrm{mg} /$ day (white tablets) from day 1 to day 11, and estradiol valerate $2 \mathrm{mg} /$ day plus cyroterone acetate $1 \mathrm{mg} /$ day (pink tablets) from day 12 to day 21, followed by a 7-day treatment-free interval.

\section{Outcome measurement}

The primary outcome of this trial is the MRS, which is widely used for evaluating the severity of aging symptoms. Eleven questions of the MRS can be classified into three categories: psychological, somatic, and urogenital. A higher score indicates worse symptoms. The primary outcome is measured at randomization and another five points $(4,12,16,24$, and 36 weeks after randomization). The secondary outcomes include the following items: Menopause-Specific Quality of Life; average hot-flash score during 24 hours; and the level of estradiol, folliclestimulating hormone, and luteinizing hormone in serum. The first two secondary outcomes are measured at randomization and $4,12,16,24$, and 36 weeks after randomization. The other secondary outcomes are measured before randomization and 12 and 24 weeks after randomization.

All patients are asked to fill in a 7-day hot flash diary before randomization and 4,12, 16, 24, and 36 weeks after randomization. After treatment, patients will be encouraged to finish these diaries by short messages, telephone calls, and emails in order to avoid selective loss to follow-up.

To exclude patients who have serious heart, liver, and kidney diseases, all participants will also receive routine tests of blood, liver function, kidney function, and electrocardiogram before randomization. Patients will also receive breast ultrasound and gynecological B ultrasound to exclude some uncovered galactophore and gynecological diseases. Meanwhile, aiming to evaluate the side effects of both methods, participates will receive the following items after the end of treatment: breast ultrasound and gynecological B ultrasound; and liver function (alanine aminotransferase, aspartate aminotransferase) and kidney function (blood urea nitrogen, serum creatinine).

This study will also estimate the expectations of participants using the credibility and expectancy questionnaire, aiming to clarify whether expectations make an impact on outcomes.

All patients in this study are required to document whether or not they take other medicine to relieve their perimenopause-related symptoms during 36 weeks, including the name and dosage of the medicine, the time and duration of taking, the reasons and the side effects of the medicine.

During the trial, all adverse events are to be recorded during treatment and the follow-up period, including bleeding, hematoma, fainting, serious pain, local infection, and so on, caused by acupuncture; and irregular vaginal bleeding, breast tenderness, headaches, nausea, and so on, caused by Climen ${ }^{\circ}$. Serious adverse events associated with the trial should be reported to the principal investigator immediately. All details will be documented, treatment options for rescue will be given at once, and appropriate action will be taken to prevent recurrence.

All acupuncturists were qualified to perform this trial after they participated in special training classes and passed the examination. The special training classes were held twice in August 2013, aiming to make all of the acupuncturists well understand all details of this trial. For instance, they were trained to use the central randomization method, to fill in the case report form, to find the correct points, to manipulate the needles, and so on. Additionally, to ensure the quality of this trial, the clinical monitors will check the processes of the trial and document the details of the processes once a month in every hospital. Moreover, monitors named by the 
principle investigator will check the accuracy and validity of the original data from the clinical centers. Regular meetings will be held to consider the recruitment rate, adverse events, protocol violations, difficulties and problems appearing during this study, and so on.

Patient drop-outs, their reasons, and their compliance will be recorded at the 36th week after randomization. All patients will be recorded until the end of this trial.

\section{Statistical analysis}

All data from this study will be analyzed by special analysts with SPSS 20.0 (IBM Corp., Armonk, NY, USA).

The tests of outcomes are based on the intention-totreat population. Sensitivity analyses are performed for the primary outcomes (MRS measured 16 weeks after randomization) by replacing missing data with multiple imputations and lost observations carried forward using SPSS 20.0. Multiple imputations are calculated using the propensity score method. The MRS is used as a dependent variable in an analysis of covariance, with the groups as random factors, and with baseline MRS and age as covariates, to account for potential baseline differences. Adjusted mean treatment effects are given alongside corresponding 95\% confidence intervals and $P$ values. The same analysis method will be performed for all secondary outcomes.

In addition to the above analysis, we will perform an exploratory analysis to describe group means over time. We will calculate summary statistics to reveal whether the two groups are changing in a similar or different fashion. A two-sided test is applied for available data, and $P<0.05$ is considered statistically significant.

There is one acupuncturist in each center to do all treatment, which probably introduces a clustering effect in this trial. We therefore calculated the intracluster correlation coefficient from the result of this trial and report the coefficient [28].

\section{Discussions}

This trial is sponsored and financially supported by the National Key Technology R\&D Program during the Twelfth Five-year Plan Period of China. The study reported in this article is only one part of this program, which is an international multicenter systematic evaluation of clinical effectiveness of acupuncture. The purposes of this international collaborative program are to prove the therapeutic effects and safety of acupuncture and to establish guidelines for clinical practice. The result of this trial is expected to provide convincing evidence that acupuncture is effective for patients with perimenopausal syndrome.

Previous RCTs show acupuncture in the treatment of symptoms of both perimenopausal and postmenopausal females has no advantage, compared with placebo needles [16-19]. Compared with no medical treatment (for example, self-caring, waiting), however, acupuncture is able to relieve symptoms quite well $[16,17,29]$. In view of controversial results, some researchers asserted that the placebo needles might have similar effects to verum acupuncture [30], and this view may underestimate the total effectiveness of verum acupuncture. We are therefore conducting a pragmatic trial that requires a positive drug as the control group to clarify an acupuncture therapeutic effect rather than a placebo needle. Moreover, we use a non-inferiority method to estimate the sample size, and only include perimenopausal patients. What we expect is to prove the therapeutic effects of acupuncture on perimenopausal syndrome, and provide another alternative treatment for perimenopausal women.

HRT has been in use for more than half a century and is regarded as a gold standard treatment for vasomotor, urogenital symptoms and their potential consequences such as sleep problems, and mood disorders [31-33]. HRT can improve the health-related quality of life through the alleviation of these symptoms. Meanwhile, HRT is beneficial to prevent aging women from osteoporosis, metabolic syndrome, and cardiovascular problems [4]. HRT is thus an ideal treatment for females with perimenopausal syndrome. Given all patients in this trial have an intact uterus, we choose estrogen plus progestin therapy to negate the risk of endometrial cancer rather than using estrogen alone, and 12-week treatment for women aged 40 to 55 should be safe according to the statement of the North American Menopause Society [4]. Furthermore, studies have shown that sequential estradiol valerate and cyroterone acetate combinations: relieve climacteric complaints via increasing serum estrogen, and provide a good menstrual cycle inducing a physiological secretory transformation of the endometrium without side effects [34]; exert a positive effect on body fat mass and distribution [35]; and may have protective effects for postmenopausal bone loss [36]. We therefore chose Climen $^{\oplus}$ as a positive drug control.

We chose the MRS as the primary outcome, which was initially developed to measure the severity of aging symptoms and their impact on health-related quality of life in the 1990s [37]. Three different dimensions of symptoms (psychological, somato-vegetative, and urogenital symptoms) are measured by 11 items, which can be easily completed by women themselves rather than physicians $[37,38]$. The total scores of MRS vary between 0 (no symptom) and 44 (highest degree of symptoms) based on the severity of symptoms; the higher scores patients obtain, the more serious symptoms they have. Since 1992 the MRS has been developed and proven to be reliable and valid for decades $[39,40]$. This selfassessment scale has the following objectives: to enable comparisons of the symptoms of aging between groups 
of women under different conditions; to compare severity of symptoms over time; and to measure changes before and after treatment [36-38]. Since the English version was published [41], 24 different languages versions are now available on the website [42]. Due to its reliability and validity, we chose the MRS as the primary outcome of this trial to assess perimenopausal syndrome. We chose Menopause-Specific Quality of Life as one of the secondary outcomes to assess the severity of these symptoms affecting patients' life quality. To measure the severity and frequency of hot flashes and sweats, participates will be required to fill hot-flash dairies for 1 week before each measurement point. The average hot-flash score during 24 hours is the 7 -day total score of severity ( 0 , none; 1 , mild; 2 , moderate; 3 , severe) $/ 7$.

One recent $\mathrm{RCT}$ found that traditional acupuncture continued relieving the vasomotor symptoms throughout the 3-month treatment period, while the sham acupuncture was tending towards stability after the first month [17]. A longer duration of treatment was hypothesized to be a superior effect between traditional and sham acupuncture. Accordingly, in order to observe a significant effectiveness of acupuncture, participants in our study will receive 3 months of treatment. Another study indicated that the effectiveness of acupuncture on perimenopausal syndrome was superior to HRT during the 6-month follow-up period [43]. Therefore, it is necessary to conduct a longer period of follow-up to obverse the continuous effects of acupuncture compared with positive drug, and also to assess the safety after treatment. Before this trial we conducted a questionnaire survey of 156 women with perimenopausal syndrome in the First Affiliated Hospital of Chengdu University of TCM to obtain the public's acceptance and understanding of acupuncture treatment for perimenopausal problems (the detail of this survey will be available soon). According to the results of the survey, previous RCTs and the opinions of experts, we designed this protocol for better treatment and follow-up arrangements.

Our study still has limitations. Firstly, the whole research period of this study is 37 weeks ( 1 week baseline, 12 weeks of treatment and 24 weeks of follow-up) and may increase the rate of patient drop-out. We therefore reduce the treatment demand after the first month of treatment and keep close contact with our patients via telephone calls, short messages, emails, and so forth, during the follow-up period, to decrease the drop-out rate. Secondly, the three centers of this study are in Chengdu, which is a capital city located in the southwest of China, and the patients we will include in this trial are mostly local residents. The population may therefore not represent the advantages of a multicenter study. Thirdly, participants assigned to the acupuncture group may have more opportunities for contact with their acupuncturists and build a good doctor-patient relationship rather than those of the control group, which may improve the therapeutic effect [44]. We will therefore keep close contact with patients in the control group by telephone, Internet, and so on, to decrease this potential bias. Meanwhile, it is difficult to blind patients after randomization due to the specificity of acupuncture. We therefore strictly conduct central randomization and appoint different people to perform therapy, measurement and statistical analysis.

Owing to a lack of solid evidence for effectiveness of acupuncture treatment on perimenopausal syndrome, this trial aims to clarify this issue by means of a multicenter randomized controlled large-scale trial.

In conclusion, the results of this trial are expected to confirm whether acupuncture is effective in relieving perimenopausal syndrome.

\section{Trial status}

This trial is currently in the recruitment phase.

\section{Abbreviations}

HRT: hormone replacement therapy; MRS: Menopause Rating Scale; $\mathrm{RCT}$ : randomized controlled trial; TCM: traditional Chinese medicine.

\section{Competing interests}

The authors declare that they have no competing interests.

\section{Authors' contributions}

$Y L, H Z, L Z, Q Z, Z S L$ and BYL participated in the conception and design of this trial, and in plans for the data. $Y \mathrm{~L}, \mathrm{HZ}$ and $\mathrm{QHZ}$ were responsible for planning to draft and revise the manuscript. $L Z$ and $Z S L$ are monitors of this study. $Q H Z$, EQQ, YW, QZ, HBZ, YZ, WS and XXZ are responsible for the recruitment and treatment of patients in each center. EQQ collects the data. All authors read this manuscript and approved the publication of this protocol.

\section{Acknowledgement}

This study is supported and financed by the National Key Technology R\&D Program during the Twelfth Five-year Plan Period of China (No. 2012BAI24B01). The authors would like to express gratitude to Prof. Chen Qiu from the Endocrinology Department of the First Affiliated Hospital of Chengdu University of TCM, Prof. Zhang Anren from the Rehabilitation Department, Zhang Jun from the TCM Department of the General Hospital of Chengdu Military Region of the Chinese People's Liberation Army, Prof. Lai Yuqin from the Gynecology Department and Prof. Liu Jun from the Acupuncture Department of Chengdu Integrated TCM \& Western Medicine Hospital who recruited the patients. They also appreciate the support and efforts from people who have been or will be included in this study.

\section{Author details}

${ }^{1}$ Chengdu University of Traditional Chinese Medicine, 37 Shi'er Qiao Road, Jinniu District, Chengdu, Sichuan 610075, PR China. ${ }^{2}$ General Hospital of Chengdu Military Region of the Chinese People's Liberation Army, 270 Tianhui Road, Rongdu Avenue, Chengdu, Sichuan 610083, PR China. ${ }^{3}$ First Affiliated Hospital of Chengdu University of Traditional Chinese Medicine, 39 Shi'er Qiao Road, Jinniu District, Chengdu, Sichuan 610072, PR China.

${ }^{4}$ Chengdu Integrated TCM \& Western Medicine Hospital, 18 North Wanxiang Road, Gaoxin District, Chengdu, Sichuan 610016, PR China. ${ }^{5}$ Guang'anmen Hospital, China Academy of Chinese Medical Sciences, No. 5 BeiXianGe Street, XiCheng District, Beijing 100053, PR China.

Received: 9 January 2014 Accepted: 15 May 2014

Published: 30 May 2014 


\section{References}

1. WHO Scientific Group on Research on the Menopause in the 1990s: Research on the Menopause in the 1990s: Report of a WHO Scientific Group, Volume 866. WHO Technical Report Series; 1996:1-107.

2. Kumari M, Stafford M, Marmot M: The menopausal transition was associated in a prospective study with decreased health functioning in women who report menopausal symptoms. J Clin Epidemiol 2005, 58:719-727.

3. Avis NE, Bromberger J, Colvin A, Hess R, Matthews KA, Ory M, Schochen M: Change in health-related quality of life over the menopausal transition in a multiethnic cohort of middle-aged women: Study of Women's Health Across the Nation. Menopause 2009, 16:860-869.

4. North American Menopause Society: The 2012 hormone therapy position statement of The North American Menopause Society. Menopause 2012, 19:257-271

5. Manson JE, Hsia J, Johnson KC, Rossouw JE, Assaf AR, Lasser NL, Trevisan M, Black HR, Heckbert SR, Detrano R, Strickland OL, Wong ND, Crouse JR, Stein E, Cushman M: Women's Health Initiative Investigators. Estrogen plus progestin and the risk of coronary heart disease. N Engl J Med 2003, 349:523-534.

6. Wassertheil-Smoller S, Hendrix SL, Limacher M, Heiss G, Kooperberg C, Baird A, Kotchen T, Curb JD, Black H, Rossouw JE, Aragaki A, Safford M, Stein E, Laowattana S, Mysiw WJ: Women's Health Initiative Investigators. Effect of estrogen plus progestin on stroke in postmenopausal women: the Women's Health Initiative: a randomized trial. JAMA 2003, 289:2673-2684.

7. Beral V: Million Women Study Collaborators: Breast cancer and hormonereplacement therapy in the Million Women Study. Lancet 2003, 362:1160-1427. A published erratum appears in Lancet.

8. Beral V, Bull D, Reeves G, Million Women Study Collaborators: Endometrial cancer and hormone-replacement therapy in the Million Women Study. Lancet 2005, 365:1543-1551.

9. Rossouw JE, Anderson GL, Prentice RZ, LaCroix AZ, Kooperberg C, Stefanick ML, Jackson RD, Beresford SA, Howard BV, Johnson KC, Kotchen JM, Ockene J, Writing Group for the Women's Health Initiative investigators: Risks and benefits of estrogen plus progestin in healthy postmenopausal women: principal results from the Women's Health Initiative randomized controlled trial. JAMA 2002, 288:321-333.

10. Kessel B, Kronenberg F: The role of complementary and alternative medicine in management of menopausal symptoms. Endocrinol Metab Clin North Am 2004, 33:717-739.

11. Newton KM, Buist DS, Keenan NL, Anderson LA, LaCroix AZ: Use of alternative therapies for menopause symptoms: results of a populationbased survey. Obstet Gynecol 2002, 100(1):18-25. A published erratum appears in Obstet Gynecol.

12. White A, Hayhoe S, Hart A, Ernest E, British Medical Acupuncture Society and Acupuncture Association of Chartered Physiotherapists: Survey of adverse events following acupuncture (SAFA): a prospective study of 32,000 consultations. Acupunct Med 2001, 19:84-92.

13. Ernst G, Strzyz H, Hagmeister H: Incidence of adverse effects during acupuncture therapy - a multicentre survey. Complement Ther Med 2003, 11:93-97.

14. NIH Consensus Conference: Acupuncture. JAMA 1998, 280:1518-1524

15. Avis $N E$, Brockwell $S$, Colvin $A: A$ universal menopausal syndrome? Am J Med 2005, 118(Suppl 12B):37-46.

16. Avis NE, Legault C, Coeytaux RR, Pian-Smith M, Shifren JL, Chen W, Valaskatgis $\mathrm{P}: \mathrm{A}$ randomized, controlled pilot study of acupuncture treatment for menopausal hot flashes. Menopause 2008, 15:1070-1078.

17. Painovich JM, Shufelt CL, Azziz R, Yang Y, Goodarzi MO, Braunstein GD, Karlan BY, Stewart PM, Merz CN: A pilot randomized, single-blind, placebocontrolled trial of traditional acupuncture for vasomotor symptoms and mechanistic pathways of menopause. Menopause 2012, 19:54-61.

18. Vincent A, Barton DL, Mandrekar JN, Cha SS, Zais T, Wahner-Roedler DL, Keppler MA, Kreitzer MJ, Loprinzi C: Acupuncture for hot flashes: a randomized, sham-controlled clinical study. Menopause 2007, 14:45-52.

19. Kim Dl, Jeong JC, Kim KH, Rho JJ, Choi MS, Yoon SH, Choi SM, Kang KW, Ahn HY, Lee MS: Acupuncture for hot flushes in perimenopausal and postmenopausal women: a randomized, sham-controlled trial. Acupunct Med 2011, 29:249-256.

20. Sturdee DW, Pines A, International Menopause Society Writing Group: Updated IMS recommendations on postmenopausal hormone therapy and preventives strategies for midlife health. Climacteric 2011, 14:302-320
21. North American Menopause Society: The role of calcium in peri- and postmenopausal women: consensus opinion of The North American Menopause Society. Menopause 2001, 8:84-95.

22. Gallicchio L, Whiteman MK, Tomic D, Miller KP, Langenberg P, Flaws JA: Type of menopause, patterns of hormone therapy use, and hot flashes. Fertil Steril 2006, 85:1432-1440.

23. Kritz-Silverstein D, Goldani Von Mühlen D, Barrett-Connor E: Prevalence and clustering of menopausal symptoms in older women by hysterectomy and oophorectomy status. J Womens Health Gend Based Med 2000, 9:747-755.

24. Pockaj BA, Loprinzi CL, Sloan JA, Novotny PJ, Barton DL, Hagenmaier A, Zhang $\mathrm{H}$, Lambert GH, Reeser KA, Wisbey JA: Pilot evaluation of black cohosh for the treatment of hot flashes in women. Cancer Invest 2004, 22:515-521.

25. Cheng J, Li ZR: A study into point selection regularity in treatment of perimenopausal syndrome with acupuncture [in Chinese]. J Nanjing University TCM 2010, 26:21-23.

26. Xin Y, Wang TF, Du CFLIL, Ren J, Jin Z, Zhao H, Jing Y, Zou XJ, Liu HQ Chen Y, Wang LN, Liu RF, Wang QG: Distribution characteristics of common syndrome types and syndrome elements extracted by experts' experience in perimenopausal and postmenopausal women [in Chinese]. J Chinese Integrative Med 2009, 7:522-566.

27. Zheng XY: Guiding Principle of Clinical Research for New Drugs of Traditional Chinese medicine [in Chinese]. Beijing, China: China Medical Science and Technology Press; 2000.

28. Campbell MK, Grimshaw JM, Elbourne DR: Intracluster correlation coefficients in cluster randomized trials: empirical insights into how should they be reported. BMC Med Res Methodol 2004, 4:9.

29. Kim KH, Kang KW, Kim DI, Kim HJ, Yoon HM, Lee JM, Jeong JC, Lee MS, Jung HJ, Choi SM: Effects of acupuncture on hot flashes in perimenopausal and postmenopausal women - a multicenter randomized clinical trial. Menopause 2010, 17:269-280

30. White P, Lewith G, Hopwood V, Prescott P: The placebo needle, is it a valid and convincing placebo for use in acupuncture trials? A randomized, single-blind, cross-over pilot trial. Pain 2003, 106:401-409.

31. National Institutes of Health: NIH state of the science conference statement on management of menopause-related symptoms. HIN Consensus State Science Statements 2005, 22:1-38.

32. Maclennan AH, Broadbent JL, Lester S, Moore V: Oral oestrogen and combined oestrogen/progestogen therapy versus placebo for hot flushes. Cochrane Database Syst Rev 2004, 4, CD002978.

33. Barnabei VM, Cochrane BB, Aragaki AK, Nygaard I, Williams RS, McGovern PG, Young RL, Wells EC, O'Sullivan MJ, Chen B, Schenken R, Johnson SR: Women's Health Initiative Investigators. Menopausal symptoms and treatment-related effects of estrogen and progestin in the Women's Health Initiative. Obstet Gynecol 2005, 105(5 Pt 1):1063-1073.

34. Koninckx PR, Lauweryns JM, Cornillie FJ: Endometrial effects during hormone replacement therapy with a sequential oestradiol valerate/ cyproterone acetate preparation. Maturitas 1993, 16:97-110.

35. Gambacciani M, Ciaponi M, Cappagli B, De Simone L, Orlandi R, Genazzani AR: Prospective evaluation of body weight and body fat distribution in early postmenopausal women with and without hormonal replacement therapy. Maturitas 2001, 39:124-132.

36. Riis BJ, Jensen J, Christiansen C: Cyproterone acetate, an alternative gestagen in postmenopausal oestrogen/gestagen therapy. Clin Endocrinol (Oxf) 1987, 26:327-334.

37. Hauser GA, Huber IC, Keller PJ, Lauritzen C, Schneider HP: Evaluation of climacteric symptoms (Menopause Rating Scale) (in German). Zentralb/ Gynakol 1994, 116:16-23.

38. Potthoff $P$, Heinemann LA, Schneider HP, Rosemeier HP, Hauser GA: The Menopause Rating Scale (MRS II): methodological standardization in the German population [in German]. Zentralbl Gynakol 2000, 122(5):280-286.

39. Schneider HP, Heinemann LA, Rosemeier HP, Potthoff P, Behre HM: The Menopause Rating Scale (MRS): reliability of scores of menopausal complaints. Climacteric 2000, 3:59-64.

40. Schneider HP, Heinemann LA, Rosemeier HP, Potthoff P, Behre HM: The Menopause Rating Scale (MRS): comparison with Kupperman index and quality-of-life scale SF-36. Climacteric 2000, 3:50-58.

41. Heinemann $L A$, Potthoff $P$, Schneider HP: International versions of the Menopause Rating Scale (MRS). Health Qual Life Outcomes 2003, 1:28.

42. MRS - The Menopause Rating Scale. http://www.menopause-rating-scale. info. 
43. Xia XH, Hu L, Qin ZY, Zhou J, Meng L, Li WL, Tian LY, Zhang YJ: Multicentral randomized controlled clinical trials about treatment of perimenopausal syndrome with electroacupuncture of sanyinjiao (SP 6) [in Chinese]. Zhen Ci Yan Jiu 2008, 33:262-266.

44. Benedetti F: Placebo and the new physiology of the doctor-patient relationship. Physiol Rev 2013, 93:1207-1246.

doi:10.1186/1745-6215-15-198

Cite this article as: Li et al:: Use acupuncture to relieve perimenopausal syndrome: study protocol of a randomized controlled trial. Trials 2014 15:198.

\section{Submit your next manuscript to BioMed Central} and take full advantage of:

- Convenient online submission

- Thorough peer review

- No space constraints or color figure charges

- Immediate publication on acceptance

- Inclusion in PubMed, CAS, Scopus and Google Scholar

- Research which is freely available for redistribution 